\title{
SALIDA, VOZ O LEALTAD. ORIENTACIONES Y FORMAS DE PARTICIPACIÓN EN AMÉRICA LATINA
}

\author{
Exit, Voice or Loyalty. Citizens' Orientations and Participation Mechanisms in \\ Latin America
}

\section{Saída, voz ou lealdade. Orientações e formas de participação na América Latina}

\author{
DEL TRONCO, José. FLACSO Sede Académica de México (México)
}

jdeltronco@flacso.edu.mx

Fecha de recibido: 13 de mayo de 2015

Fecha de aceptado: 1 de junio de 2015

\section{RESUMEN}

En América Latina, el desencanto de los ciudadanos con las instituciones representativas de gobierno ha sido evidenciado por una multiplicidad de trabajos a lo largo de las últimas dos décadas. Este desaliento ha sido provocado por la baja calidad de los procesos gubernativos de las democracias latinoamericanas. Escándalos de corrupción, elecciones sospechadas, decisiones no representativas de los intereses ciudadanos y problemas estructurales no resueltos como la pobreza o la desigualdad ayudan a explicar las actitudes generalizadas de desconfianza institucional. Esta constatación ha reflotado la discusión sobre la importancia de contar con mecanismos que favorezcan una mayor participación de los ciudadanos en los procesos de toma de decisiones, incluyendo la asistencia periódica a las urnas. Sin embargo, poco sabemos acerca del perfil de aquellos que ante una caída en la calidad de los procesos de gobierno deciden participar - ya sea de manera propositiva, ya de manera directa- así como de quienes deciden 'salir'; es decir, abstenerse de participar en los asuntos públicos. Partiendo del trabajo seminal de Albert Hirschman (1977), y partir del análisis de datos de opinión pública de la onda 2008 del Latinobarometro, el 
presente trabajo se propone responder a estos interrogantes, así como discutir las implicaciones de las respuestas derivadas de la evidencia.

Palabras clave: capacidad gubernativa, participación política, perfiles ciudadanos, América Latina.

\begin{abstract}
In Latin America, citizen 's discontent with representative institutions has been shown by many scholars during last two decades. Latin American citizens express a general sentiment of disengagement and distrust from politics and politicians. This fact has re-launched the debate on the convenience of social participation in the policy-making process, beyond and complementing the electoral mechanism. Though, there is a lack of evidence to establish under what conditions Latin American citizens decide (or not) to participate in public affairs to contribute to solve those problems. Theoretically based on the seminal work of Albert Hirschman (1977), the current study uses public opinion data from Latinbarometer (2008) to find the factors that increase (voice) and decrease (exit) the probability of citizens to participate in collective actions.
\end{abstract}

Keywords: Governing capacity, political participation, citizens profiles, Latin America.

\title{
RESUMO
}

Na América Latina, o desencanto dos cidadãos com as instituições representativas de governo tem sido evidenciado por uma multiplicidade de trabalhos ao longo das últimas duas décadas. Este desalento tem sido provocado pela baixa qualidade dos processos governativos das democracias latino-americanas. Escândalos de corrupção, eleições suspeitadas, decisões não representativas dos interesses cidadãos e problemas estruturais não resolvido como a pobreza ou a desigualdade ajudam a explicar as atitudes generalizadas de desconfiança institucional. Esta constatação tem ressuscitado a discussão sobre a importância de contar com mecanismos que favoreçam uma maior participação dos cidadãos nos processos de tomada de decisões, incluindo a assistência periódica às urnas. No entanto, pouco sabemos acerca do perfil daqueles que ante uma caída na qualidade dos processos de governo decidem participar -seja de maneira propositiva, seja de maneira direta- assim como de quem decidem 'sair'; é dizer, se abster de participar nos assuntos públicos. Partindo do trabalho seminal de Albert Hirschman (1 977), e partir da análise de dados de opinião pública da onda 2008 do Latino-barômetro, o presente trabalho propõe-se responder a estes interrogantes, assim como discutir as implicações das respostas derivadas da evidência.

Palavras-chave: Capacidade governativa, participação política, perfiles cidadãos, América Latina. 


\section{Gobierno representativo y actitudes ciudadanas}

Uno de los rasgos más salientes de las democracias en América Latina desde la transición es el desencanto de los ciudadanos con las instituciones representativas de gobierno. De acuerdo con datos del Latinobarómetro, entre 1996 y 2013, dos terceras partes de los latinoamericanos confían poco o nada en instituciones como el congreso o los partidos políticos y tres de cada cuatro considera que los gobiernos no representan los intereses del pueblo, sino los de una minoría poderosa.

Esta constatación ha reflotado la discusión sobre la importancia de contar con mecanismos que favorezcan una mayor participación de los ciudadanos en los procesos de toma de decisiones, más allá de la asistencia periódica a las urnas (Isunza y Gurza, 2010; Altman, 2010; Welp, 2011). El argumento sostiene que la existencia de más, y más canales directos de interlocución entre la sociedad y el sistema político redundarán en decisiones más eficaces para satisfacer los intereses de los actores involucrados en el problema público que se pretende atender (Buchanan y Tullock, 1980).

Sin embargo, la participación política, ya sea en forma de activación de la voz de protesta o a través de la búsqueda de soluciones ciudadanas para los problemas públicos, requiere de ciertas condiciones, tanto a nivel individual como del sistema político-institucional (Almond y Verba, 1959; Milbraith y Goel, 1965; Putnam, 1993; Offe, 2006). La cultura política de la sociedad, las disposiciones constitucionales, las estrategias de movilización política y los atributos individuales - personales y relacionales- de los ciudadanos, son factores que favorecen u obstaculizan el surgimiento de espacios autónomos, institucionalizados y efectivos para la participación de la sociedad en los procesos de toma de decisiones (De Sousa, 2004; Vecchione y Caprara, 2009; Irvin, 2006; Best y Krueger, 2005; Uslaner, 2002).

Durante la última década, en particular, la literatura especializada ha destacado el surgimiento de nuevas formas de participación o 'innovaciones democráticas' (Avritzer, 2010; Zaremberg, 2012). Sin embargo, queda mucho por conocer en torno a la propensión de los ciudadanos a participar -de manera individual o colectiva-, en los asuntos públicos en general, y a través de estos mecanismos, en particular. ¿Son los ciudadanos más descontentos los más propensos a participar? ¿Si la participación implica un costo, quiénes son los que están dispuestos a asumirlo? ¿Aquellos a quienes más afecta la caída de la capacidad gubernativa, porque tienen menos recursos propios para hacer frente a la satisfacción de necesidades, o quiénes tienen mayor capacidad de incidencia en las decisiones del Gobierno, aunque puedan sustituir bienes públicos de baja calidad por bienes privados en el mercado? En definitiva, el artículo intenta develar: i) si quienes están más interesados en cambiar el statu quo - por ser los más descontentos-, son quienes más participan. ii) Si hay alguna relación entre la orientación (voz, lealtad) y las formas de participación (propositivas o directas) elegidas por los distintos perfiles de ciudadanos.

Recuperando la propuesta de Hirschman, el presente trabajo se propone indagar cuáles son los perfiles más dispuestos a activar su voz de protesta ante un descenso en la eficacia de los gobiernos para lidiar con los problemas económicos sociales actuales (voice); cuáles son aquellos que, por diversos motivos, mantienen su lealtad (loyalty) y cuáles deciden, ante un cálculo de los costos y beneficios de la participación, 'salirse' del juego político democrático y mantenerse al margen de toda forma de participación (exit).

El documento se estructura así: en un primer momento, se evidencia brevemente el desencanto de los ciudadanos latinoamericanos con el funcionamiento de la democracia representativa, y se presentan los argumentos 
utilizados para justificar - desde una perspectiva de política pública- por qué la participación social podría modificar esta situación. Seguidamente, se presentan las condiciones que, de acuerdo con la literatura especializada, pueden favorecer una mayor incidencia de los ciudadanos en el proceso de toma de decisiones, destacándose los atributos individuales como objeto del presente estudio. A continuación, y a partir de datos de opinión pública, se analiza en qué medida los ciudadanos de la región latinoamericana cumplen con estas características y cómo se relaciona cada una de ellas con los distintos tipos de participación política. En el final, se presentan algunas conclusiones así como las implicaciones de estos hallazgos para el futuro de las estrategias participativas en el continente.

\section{Representación política y decepción}

En cualquiera de sus formas, la participación política es un elemento fundamental de un sistema democrático (Dahl, 1989). Ya sea a través de la elección de representantes o mediante la participación directa en la toma de decisiones, un sistema político democrático debe asegurar la conexión entre las preferencias e intereses de los ciudadanos y el proceso de formulación de políticas (Anduiza y Bosch, 2004).

En las democracias contemporáneas, dicha conexión se intenta garantizar a través de la elección de mandatarios, cuya responsabilidad es hablar en nombre de los intereses de sus mandantes (Pitkin, 1985). En tal sentido, los representantes son responsables de sus decisiones y de sus resultados frente a los electores. Esto ocurre, básicamente, en el momento de las elecciones (Schedler, 2007).

En este proceso de negociación y debate entre representantes de diversos intereses y posturas ideológicas, los intermediarios - partidos, parlamento, la clase política en su conjunto- son actores fundamentales. De ellos depende que la deliberación y formulación de políticas respondan a las necesidades de los ciudadanos, y/o que se resuelvan los problemas públicos ingresados a la agenda de gobierno (Easton, 1953; Aguilar, 1992).

En América Latina, esta concepción elitista del proceso de representación según la cual los ciudadanos se limitan a elegir a sus representantes para que sean estos los responsables de formular, implementar y evaluar las políticas públicas, ha mostrado también sus limitaciones. La realidad de las democracias representativas ${ }^{1}$ contemporáneas muestra que las instituciones políticas (parlamentos y partidos políticos, en particular) están en crisis, que los ciudadanos consideran que los gobernantes no representan los intereses del pueblo y que, cuando ello sucede, la confianza en los gobiernos disminuye entre la opinión pública.

1 El término democracia representativa hace referencia a un régimen político en el cual "el pueblo no delibera ni gobierna sino a través de sus representantes", si bien estos últimos son electos a través del voto popular. Para una interesante discusión sobre la compatibilidad del régimen democrático y el gobierno representativo, ver Manin (1998). 


\section{Gráfica 1. Percepción de representatividad y actitudes hacia la democracia} en México y Uruguay (2004-2013)
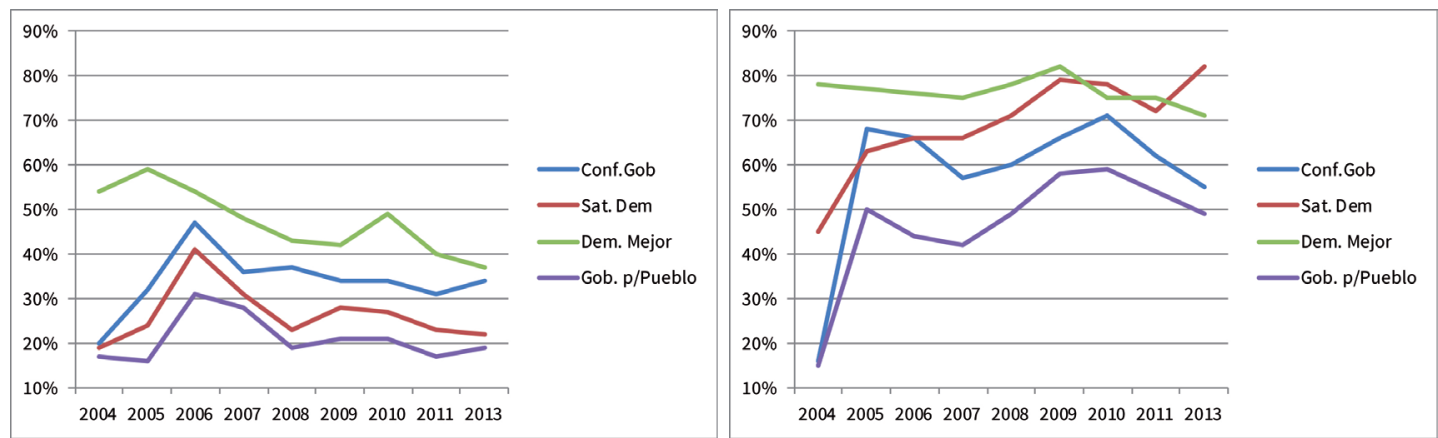

Fuente: elaboración propia con base en el Latinobarómetro.

La gráfica 1 muestra que aún en dos países con tradiciones y experiencias democráticas muy disímiles como México y Uruguay, la consideración del nivel de representatividad del gobierno se relaciona con las actitudes hacia la democracia. La satisfacción con el funcionamiento de la democracia, la confianza en el gobierno e incluso la valoración normativa del régimen están relacionadas positivamente con la capacidad de las autoridades electas de representar los intereses o satisfacer las necesidades de los ciudadanos.

El valor de esta información para el presente análisis radica en que los ciudadanos valoran positivamente que sus representantes actúen en su nombre, y cuando ello no ocurre, su grado de confianza en las instituciones democráticas disminuye.

Pero ¿qué ocurre cuando los gobiernos no cumplen con los mandatos, no representan los intereses de sus ciudadanos, fracasan en el proceso de provisión de bienes públicos o no generan las condiciones para el desarrollo individual y social de sus ciudadanos? Según Hirschman, la respuesta de los consumidores (en el mercado) o de los ciudadanos (en el espacio público) ante una caída en la calidad de los bienes producidos (por los agentes económicos o por los gobiernos) puede adquirir tres modalidades: i) la 'salida', representada por el comportamiento de aquellos que deciden sustituir los bienes - cuya calidad ha bajado- o abstenerse de su consumo. ii) La 'voz', que es la acción política por excelencia, implica el reclamo, la protesta y la expresión de descontento frente a la decepción (Hirschman, 1986) generada por una caída inesperada en la calidad del bien a consumir. iii) La 'lealtad' es la tercera posible reacción, caracterizada por la estabilidad en el consumo de aquellos bienes producidos, ahora, con una calidad inferior. En el caso de la participación política, esto implicaría no solo seguir participando sino hacerlo a favor del gobierno cuya capacidad de provisión de bienes públicos de calidad (justicia, seguridad, educación, salud) ha disminuido.

\section{La participación ciudadana y sus determinantes}

De acuerdo con distintas versiones de la teoría democrática (Dahl, 1989; Avritzer, 2010; Peruzzotti y Smoluwitz, 2002; Cleary y Stokes, 2006; Isunza y Gurza, 2010), el control social de las decisiones de gobierno es fundamental 
para garantizar o al menos aumentar la probabilidad de que las políticas públicas satisfagan de la mejor manera las preferencias ciudadanas. Si bien, tales postulados no son más que una hipótesis, en ello radica que los mecanismos de participación ciudadana sean considerados crecientemente como una opción deseable para mejorar la representatividad y, por tanto, la orientación pública de las políticas (Subirats, 2009; Parés, 2009). Por ello, responder a la preguntas quiénes participan, cómo y con qué frecuencia parece de gran relevancia no solo en términos teóricos sino también para entender el funcionamiento de la democracia en el continente, más allá de los abordajes clásicos de la transición (Anduiza y Bosch, 2004).

El enfoque participativo de las políticas públicas se contrapone con el abordaje tradicional, entendido, este último, como un modo jerárquico de toma de decisiones en el que los funcionarios de gobierno privilegian una supuesta racionalidad de las políticas por sobre las preferencias 'subóptimas' de los actores sociales afectados por el problema público que se pretende resolver (Lasswell, 1992).

Sin embargo, como señala Parés (2009), resulta difícil encontrar una definición universalmente aceptada de 'participación social' en políticas públicas, para darle consistencia a este nuevo enfoque. Por un lado, se encuentran las definiciones que entienden por participación a "todas aquellas prácticas políticas y sociales a través de las cuales la ciudadanía pretende -y tiene capacidad de - incidir efectivamente sobre la toma de decisiones públicas". En tal sentido, la participación social refiere tanto al voto ciudadano en las elecciones como a las distintas formas de acción colectiva de apoyo, protesta o reivindicación social.

Alternativamente, la participación social es concebida como todos aquellos actos de presencia de ciudadanos o grupos civiles organizados en un proceso de toma de decisiones públicas (Vargas, 2011), más allá de su consecuencia sobre el proceso decisorio. En el primer caso, la participación implica cierta incidencia sobre el proceso, mientras que en este, la mera presencia de los actores no gubernamentales es condición suficiente para ser considerada una experiencia participativa.

Finalmente, nos encontramos con trabajos que diferencian la participación social de la que tiene lugar durante las elecciones, diseñadas para elegir representantes que decidirán en nombre de los ciudadanos (Isunza y Gurza, 2010). Para estos autores, la participación social en políticas públicas se da a partir de la existencia de interfases socio-estatales, que constituyen espacios relativamente institucionalizados de encuentro entre los ciudadanos y los actores gubernamentales. Estos espacios están concebidos para involucrar directamente a los gobernados en los procesos de toma de decisión, y pueden ser meramente consultivos o tener carácter mandatorio².

De tal manera, e independientemente del formato e intensidad que asuma la participación de los ciudadanos en la toma de decisiones, existe un elemento común a las distintas definiciones que son "las acciones voluntarias ${ }^{3}$ de los ciudadanos con el propósito de incidir directamente en las políticas gubernamentales (Barnes y Kaase, 1979; Parés, 2009) y/o en la resolución de problemas colectivos". De acuerdo con esta definición, la voluntad de incidir de manera directa sobre las decisiones colectivas y la autonomía de los actores que la expresan constituyen, entonces, los criterios mínimos a considerar, para distinguir a la participación ciudadana de fenómenos relacionados, pero diferentes.

2 Incluimos aquí los mecanismos (electorales) de democracia directa (MDD) que permiten conocer la opinión de los ciudadanos acerca de algún asunto de política pública y en algunos casos, vincular esta voz ciudadana con las decisiones gubernamentales.

3 Independiente de todo tipo de presión o inducción por parte de algún 'broker' partidista o gubernamental. 


\section{La participación ciudadana y sus condiciones}

La propensión de los ciudadanos a participar en asuntos públicos puede ser estudiada desde diferentes disciplinas y enfoques (Irving, 2006). Para quienes abordan el problema desde la psicología, lo central es el estudio de la estructura de personalidad. Desde esta perspectiva, el factor que mejor explica la propensión de un individuo a involucrarse en política es su "percepción de eficacia interna". Distintos autores han demostrado que aquellos individuos que se consideran incapaces de 'tomar el control' en determinadas situaciones de su vida, se perciben también con menor capacidad para influir efectivamente en el proceso político, disminuyendo su interés en los asuntos públicos (Bandura, 1986; Vecchione y Caprana, 2009).

Por su parte, para quienes analizan el impacto de las características sociales de los individuos, la adscripción de clase, el nivel educativo, o la disponibilidad de recursos (simbólicos o materiales) son los factores más destacados como condiciones que favorecen la participación política de los ciudadanos (Verba, Lehman y Brady, 1995). Muchos de estos estudios han mostrado una asociación significativa entre los recursos socioeconómicos ${ }^{4}$ y la propensión a participar. Sin embargo, los mecanismos causales a través de los cuales los primeros influyen sobre la segunda no están del todo claros (Ruedin, 2007)

Desde una perspectiva racional, diferentes autores sostienen que la participación política de los ciudadanos responde a la existencia de incentivos selectivos (Olson, 1965). En ausencia de beneficios particularistas, los ciudadanos no tendrían razones - más allá de las vinculadas con su ética política (Bleu, 2000) - para participar en una actividad que genera costos, y cuyos beneficios son más bien difusos y de mediano plazo. Sin embargo, la evidencia demuestra que los ciudadanos (y en especial aquellos de quienes menos se esperaría: los pobres, las mujeres o los menos educados) sí participan en consejos consultivos, redes de políticas y movimientos sociales, a favor de causas de distinto tipo (Hawkins, 2010). En este caso, la explicación es la existencia de beneficios particulares y de corto plazo (a veces vinculadas con esquemas clientelares) que modifican la ecuación de los ciudadanos y los motivan -de manera más o menos interesada- a participar de acciones colectivas (Brusco, Nazareno y Stokes, 2004).

Vinculada con esta última, aunque desde un enfoque culturalista, la teoría del capital social, sostiene que aquellos que establecen una mayor cantidad de lazos con los miembros de su comunidad, son más propensos a participar en asuntos públicos. La identificación con el lugar así como con el bienestar de quienes allí viven, motivan a los ciudadanos a participar en decisiones colectivas (Ruedin, 2007). La confianza generalizada y la existencia de redes para la consecución de bienes públicos son, desde esta perspectiva, los factores que explican la mayor disposición de los ciudadanos a participar en acciones colectivas y, adicionalmente, el buen funcionamiento de los gobiernos (Putnam, 1993).

Entre las perspectivas que hacen mayor hincapié en la estructura que en los agentes, una primera aproximación es la institucional. Schmitter y Karl (1991) sostienen, por ejemplo, que la democratización de los sistemas políticos contribuye al surgimiento de actitudes cívicas favorables a la participación ciudadana. Contrariamente, el enfoque culturalista postula que los cambios institucionales son el resultado de la cultura política prevaleciente

4 El modelo SES intenta explicar la participación política de los ciudadanos a partir de recursos como el nivel de ingreso, la clase social de adscripción y/o las competencias cívicas de los individuos. 
en una sociedad. (Almond y Verba, 1959; Granato, Inglehart y Leblang, 1996). Para estos autores, la extensión de valores cívicos y democráticos entre la población es lo que explica su propensión a participar políticamente y como consecuencia de ello, la democratización de los sistemas políticos.

Finalmente, los estudios poblacionales y demográficos afirman que la pertenencia del individuo a un determinado cohorte generacional condiciona la disposición de los ciudadanos a participar políticamente. Quienes forman parte de generaciones cuyos miembros, al ingresar a la vida pública, comparten un interés generalizado por los asuntos públicos, serán individuos con mayor propensión a participar políticamente y viceversa. (Castillo, 2008; Franklin, Lyons y Marsh, 2004).

Como ya fue mencionado, el presente estudio tiene dos objetivos: por un lado, entender cuáles son las condiciones bajo las cuales los ciudadanos se deciden a participar políticamente, ya sea de acciones colectivas (trabajar por la comunidad) o de acciones individuales (voto), respecto de aquellos que prefieren la 'salida'. En segundo término, intentaremos determinar bajo qué condiciones un ciudadano que decide participar, lo hace de manera favorable al gobierno de turno, expresando su lealtad, y bajo qué otras se decide a activar su 'voz' de protesta, expresando su oposición. A partir de datos de opinión pública del Latinobarómetro 2008 (que permite hacerlo por incluir una batería de preguntas específicas sobre estos temas) ${ }^{5}$ se ofrece una panorama sobre la medida en que los ciudadanos -a nivel individual- cuentan o no con recursos, valores actitudes e incentivos que favorecen u obstaculizan su vinculación con el sistema político.

\section{Evidencia empírica: participantes, orientaciones y formas de participación}

En este apartado se analizan dos formas o tipos de participación: la propositiva y la directa. La primera refiere a las actividades legales a través de las cuales los ciudadanos solicitan y/u orientan la intervención de las autoridades en determinados asuntos, ya sea para cambiar o para mantener el statu quo. En este grupo, presentamos dos categorías o formas de participación: firmar peticiones y votar.

Por su parte, la participación de tipo directo engloba a todas aquellas actividades que implican una intervención directa por parte de los ciudadanos, ya sea en la solución de problemas colectivos, o en la defensa de intereses de determinados grupos. Aquí, analizamos las dos más frecuentes de todas las incluidas en el cuestionario de Latinobarómetro: Trabajar por el barrio o la comunidad y trabajar por la mejora del medioambiente.

Al analizar esta distinción de formas de participación ciudadana, es bastante llamativo el hecho de que las actividades propositivas parecen tener un carácter más político - formalmente hablando - que las directas. Tanto 'realizar peticiones a las autoridades' o 'votar' son actividades que tienen una clara intención de incidir sobre la voluntad de quienes toman decisiones en el Gobierno, mientras que el trabajo comunitario, o el trabajo para la mejora del medioambiente, suelen ser actividades que impactan directamente sobre un aspecto más acotado de la realidad, que -si bien están orientadas a resolver un problema social o colectivo- no refieren necesariamente a una intervención del sistema político.

5 Dado que no se incluyen aquí los factores estructurales -especialmente institucionales e históricos-a considerar en un estudio exhaustivo sobre los determinantes de la participación ciudadana, las conclusiones son preliminares y restringidas a las variables aquí analizadas. 
Tabla 1. Formas de participación y tipo de conductas implicadas. América Latina 2008

\begin{tabular}{|c|c|c|c|c|c|c|c|}
\hline \multirow{3}{*}{ País } & \multirow{3}{*}{$\begin{array}{c}\text { Se } \\
\text { abstendrían } \\
\text { de votar }\end{array}$} & \multicolumn{3}{|c|}{ Formas propositivas } & \multicolumn{3}{|c|}{ Formas directas } \\
\hline & & \multicolumn{2}{|c|}{ Votarian por } & \multirow{2}{*}{$\begin{array}{c}\text { Han firmado } \\
\text { peticiones }\end{array}$} & \multirow{2}{*}{$\begin{array}{l}\text { Han trabajado } \\
\text { por el barrio o la } \\
\text { comunidad }\end{array}$} & \multicolumn{2}{|c|}{$\begin{array}{l}\text { Buscan mejorar el } \\
\text { medioambiente con }\end{array}$} \\
\hline & & Gobierno & Oposición & & & Trabajo & Dinero \\
\hline Argentina & $37,81 \%$ & $36,94 \%$ & $25,25 \%$ & $32,40 \%$ & $20,90 \%$ & $16,90 \%$ & $9,60 \%$ \\
\hline Bolivia & $34,47 \%$ & $54,87 \%$ & $10,66 \%$ & $13,70 \%$ & $31,90 \%$ & $20,70 \%$ & $18,90 \%$ \\
\hline Brasil & $13,62 \%$ & $42,37 \%$ & $44,02 \%$ & $13,30 \%$ & $23,80 \%$ & $32,00 \%$ & $13,80 \%$ \\
\hline Colombia & $21,05 \%$ & $30,01 \%$ & $48,94 \%$ & $25,00 \%$ & $20,30 \%$ & $33,40 \%$ & $17,50 \%$ \\
\hline Costa Rica & $37,61 \%$ & $33,05 \%$ & $29,34 \%$ & $14,30 \%$ & $26,00 \%$ & $27,60 \%$ & $14,00 \%$ \\
\hline Chile & $22,44 \%$ & $24,68 \%$ & $52,08 \%$ & $14,50 \%$ & $14,20 \%$ & $10,60 \%$ & $9,70 \%$ \\
\hline Ecuador & $24,46 \%$ & $56,98 \%$ & $18,57 \%$ & $11,80 \%$ & $24,50 \%$ & $20,30 \%$ & $12,10 \%$ \\
\hline El Salvador & $14,22 \%$ & $35,96 \%$ & $50,73 \%$ & $12,60 \%$ & $14,90 \%$ & $7,10 \%$ & $25,40 \%$ \\
\hline Guatemala & $41,41 \%$ & $23,16 \%$ & $34,42 \%$ & $16,90 \%$ & $27,70 \%$ & $24,30 \%$ & $27,30 \%$ \\
\hline Honduras & $25,36 \%$ & $36,72 \%$ & $37,92 \%$ & $16,80 \%$ & $40,50 \%$ & $33,30 \%$ & $21,30 \%$ \\
\hline México & $20,94 \%$ & $32,93 \%$ & $46,13 \%$ & $24,70 \%$ & $28,80 \%$ & $46,70 \%$ & $12,80 \%$ \\
\hline Nicaragua & $26,02 \%$ & $38,01 \%$ & $35,96 \%$ & $14,00 \%$ & $25,90 \%$ & $23,80 \%$ & $14,30 \%$ \\
\hline Panamá & $21,36 \%$ & $40,54 \%$ & $38,10 \%$ & $18,20 \%$ & $20,70 \%$ & $13,40 \%$ & $13,20 \%$ \\
\hline Paraguay & $16,24 \%$ & $24,71 \%$ & $59,06 \%$ & $14,50 \%$ & $42,50 \%$ & $34,40 \%$ & $8,80 \%$ \\
\hline Perú & $38,09 \%$ & $7,35 \%$ & $54,56 \%$ & $18,90 \%$ & $25,10 \%$ & $21,00 \%$ & $17,10 \%$ \\
\hline Uruguay & $9,20 \%$ & $55,08 \%$ & $35,71 \%$ & $35,90 \%$ & $14,60 \%$ & $15,50 \%$ & $8,50 \%$ \\
\hline Venezuela & $15,54 \%$ & $51,42 \%$ & $33,03 \%$ & $22,40 \%$ & $27,80 \%$ & $28,60 \%$ & $22,00 \%$ \\
\hline Rep. Dom. & $18,76 \%$ & $48,37 \%$ & $32,87 \%$ & $11,90 \%$ & $27,00 \%$ & $10,80 \%$ & $33,60 \%$ \\
\hline Total & $23,93 \%$ & $37,78 \%$ & $38,29 \%$ & $18,40 \%$ & $25,40 \%$ & $23,30 \%$ & $16,70 \%$ \\
\hline $\mathrm{N}$ & \multicolumn{3}{|c|}{13610} & 21000 & 14808 & \multicolumn{2}{|c|}{16327} \\
\hline Conducta & Salida & Lealtad & \multicolumn{2}{|c|}{ Voz } & \multicolumn{3}{|c|}{ ¿Salida, voz o lealtad? } \\
\hline
\end{tabular}

Fuente: elaboración propia con base en el Latinobarómetro 2008.

De acuerdo con los resultados de la tabla 1, los latinoamericanos participan más de forma directa, que a través de estrategias propositivas, excepto cuando se trata de votar (75\%). Entre las formas no electorales de participación, uno de cada cuatro latinoamericanos dice trabajar por el barrio o la comunidad o por la mejora del medioambiente, mientras que en promedio menos de una quinta parte del total dice haber firmado alguna vez una petición para presentar a las autoridades. Por su parte, un 23,9\% dice no recurrir a forma alguna de participación política o social. Esto es, uno de cada cuatro ciudadanos de la región (con un máximo del 41 \% en Guatemala y un mínimo del $9 \%$ en Uruguay), se mantiene al margen de toda forma de acción colectiva.

6 Si las elecciones fueran el próximo domingo. 
Si analizamos la frecuencia de la participación ciudadana por países, podemos notar que:

- Los dos países con mayores porcentajes de participación en estrategias propositivas - firmar peticiones y asistir a manifestaciones - son a su vez dos de los cuatro con una menor proporción de ciudadanos participando en acciones - directas- (Argentina y Uruguay).

- Hay países como México y Venezuela que están entre los más participativos en ambos rankings, mientras que otros como Chile y Ecuador cuentan con un porcentaje muy bajo de ciudadanos participando en ambos tipos de estrategias.

- Los países que cuentan con mayor número de ciudadanos, participando en acciones directas, son países con niveles de calidad institucional medios o bajos, de acuerdo a los indicadores más utilizados por la literatura especializada (Gunitzky, 2013; Levine \& Molina, 2007), mientras que en el caso de las formas de participación propositiva, el panorama es más heterogéneo.

Ahora bien, ¿cuáles son los factores que condicionan la propensión a participar en asuntos públicos, y, específicamente, en cada una de las estrategias de participación aquí presentadas? ¿Qué rasgos caracterizan a quienes participan manifestando su apoyo al Gobierno? ¿Quiénes activan su voz de protesta y quiénes deciden salirse de la acción colectiva democrática o comunitaria? Para responder a estas preguntas, se construyeron cuatro modelos de análisis, destinado cada uno de ellos a explorar los patrones culturales, sociodemográficos y políticos que aumentan la probabilidad de un ciudadano latinoamericano a asumir las distintas formas de comportamiento político: la participación a favor del Gobierno (lealtad); la participación opositora (voz); la abstención de participar (salida), y la participación directa, que promueve la mejora de condiciones sociales y comunitarias, pero que no interpela abiertamente al sistema político.

\section{Tabla 2. Formas de participación y factores explicativos (AL, 2008)}

\begin{tabular}{|c|c|c|c|c|c|c|}
\hline \multicolumn{7}{|c|}{ Modelo Logit Multinominal } \\
\hline & \multicolumn{2}{|c|}{ Votar } & \multirow{2}{*}{$\begin{array}{c}\begin{array}{c}\text { Firmar una } \\
\text { petición }\end{array} \\
\text { Lo he } \\
\text { realizado }\end{array}$} & \multirow{2}{*}{$\begin{array}{c}\text { Hacer cosas por el } \\
\text { barrio o comunidad } \\
\text { Trabajo }\end{array}$} & \multicolumn{2}{|c|}{$\begin{array}{l}\text { Mejorar el medio } \\
\text { ambiente }\end{array}$} \\
\hline Factores explicativos & $\begin{array}{l}\text { Partido de } \\
\text { Gobierno }\end{array}$ & $\begin{array}{l}\text { Partido de } \\
\text { Oposición }\end{array}$ & & & Trabajo & Donar \\
\hline \multicolumn{7}{|c|}{ p13st Valores democráticos } \\
\hline $\begin{array}{l}\text { A la gente como uno, nos da lo } \\
\text { mismo un régimen democrático } \\
\text { que uno no democrático }\end{array}$ & & & $0,6^{\star \star \star}$ & & & $0,7^{\star \star}$ \\
\hline $\begin{array}{l}\text { La democracia es preferible a cual- } \\
\text { quier otra forma de gobierno }\end{array}$ & & & & & & $0,7^{\star \star \star}$ \\
\hline \multicolumn{7}{|c|}{ p21wvsst Confianza interpersonal } \\
\hline $\begin{array}{l}\text { Se puede confiar en la mayoría de } \\
\text { las personas }\end{array}$ & 1,1 & $1,2^{\star \star}$ & $0,9^{\star \star}$ & $0,9^{\star \star}$ & & $1,4^{\star \star \star}$ \\
\hline \multicolumn{7}{|c|}{ P25st Gobierno } \\
\hline Para el bien de todo el pueblo & $2,0^{\star \star \star}$ & $1,2^{\star \star}$ & & & & \\
\hline
\end{tabular}




\begin{tabular}{|c|c|c|c|c|c|c|}
\hline \multicolumn{7}{|c|}{ Modelo Logit Multinominal } \\
\hline \multirow[b]{2}{*}{ Factores explicativos } & \multicolumn{2}{|c|}{ Votar } & \multirow{2}{*}{$\begin{array}{c}\begin{array}{c}\text { Firmar una } \\
\text { petición }\end{array} \\
\text { Lo he } \\
\text { realizado }\end{array}$} & \multirow{2}{*}{$\begin{array}{c}\text { Hacer cosas por el } \\
\text { barrio o comunidad } \\
\text { Trabajo }\end{array}$} & \multicolumn{2}{|c|}{$\begin{array}{c}\text { Mejorar el medio } \\
\text { ambiente }\end{array}$} \\
\hline & $\begin{array}{l}\text { Partido de } \\
\text { Gobierno }\end{array}$ & $\begin{array}{l}\text { Partido de } \\
\text { Oposición }\end{array}$ & & & Trabajo & Donar \\
\hline \multicolumn{7}{|c|}{ p53st Dígame, ¿votó usted en las últimas elecciones presidenciales? } \\
\hline Sí & $2,7^{\star \star \star}$ & $2,4^{\star \star \star}$ & $1,2^{\star \star}$ & $1,1^{\star}$ & & \\
\hline \multicolumn{7}{|c|}{ P56st Autoposicionamiento ideológico } \\
\hline Centro izquierda $(3,4)$ & $2,0^{\star \star \star}$ & $1,6^{\star \star \star}$ & $1,2^{\star \star \star}$ & $1,2^{\star \star \star}$ & & \\
\hline Izquierda $(0,1,2)$ & $2,2^{\star \star \star}$ & $1,4^{\star \star \star}$ & & & & \\
\hline Centro derecha $(6,7)$ & $1,9^{\star \star \star}$ & $1,9^{\star \star \star}$ & & $1,3^{\star \star \star}$ & 1,1 & $1,4^{\star \star *}$ \\
\hline Derecha $(8,9,10)$ & $2,5^{\star \star \star}$ & $2,5^{\star \star \star}$ & & $1,2^{\star \star}$ & $1,2^{\star \star \star}$ & \\
\hline \multicolumn{7}{|c|}{ P80sta Miembro organización política } \\
\hline Sí & $1,5^{\star \star \star}$ & $1,8^{\star \star \star}$ & $1,4^{\star \star \star}$ & $1,8^{\star \star \star}$ & $1,3^{\star \star \star}$ & $1,7^{\star \star \star}$ \\
\hline \multicolumn{7}{|c|}{ P80stb Miembro organización sindical } \\
\hline Sí & $1,3^{\star \star}$ & & $1,3^{\star \star \star}$ & $1,6^{\star \star \star}$ & $1,6^{\star \star \star}$ & $1,4^{\star \star}$ \\
\hline \multicolumn{7}{|c|}{ P80ste Miembro organización comunal } \\
\hline Sí & $1,3^{\star \star}$ & $1,2^{*}$ & $1,4^{\star \star \star}$ & $3,9^{\star \star \star}$ & $1,8^{\star \star \star}$ & $1,5^{\star \star *}$ \\
\hline \multicolumn{7}{|c|}{ P80STJ Miembro organización ecológica } \\
\hline Sí & & & $1,4^{\star \star \star}$ & $1,9^{\star \star \star}$ & $4,0^{\star \star \star}$ & $2,5^{\star \star \star}$ \\
\hline \multicolumn{7}{|c|}{ P81st Lo más efectivo para influir en cambiar las cosas } \\
\hline $\begin{array}{l}\text { No es posible influir para que las } \\
\text { cosas cambien, da igual lo que uno } \\
\text { haga }\end{array}$ & & & & & $1,4^{\star \star}$ & \\
\hline $\begin{array}{l}\text { Participar en movimientos de } \\
\text { protesta y exigir los cambios direc- } \\
\text { tamente }\end{array}$ & & & $1,8^{\star \star \star}$ & & $1,3^{\star *}$ & 1,2 \\
\hline $\begin{array}{l}\text { Votar para elegir a los que defienden } \\
\text { mi posición }\end{array}$ & $1,6^{\star \star \star}$ & & $1,8^{\star \star \star}$ & & & $0,7^{\star}$ \\
\hline \multicolumn{7}{|c|}{ P83st_b ¿Ha contactado a un funcionario representante municipal? } \\
\hline Lo podría realizar & & & $2,8^{\star \star \star}$ & $2,0^{\star \star \star}$ & $1,2^{\star \star \star}$ & $1,6^{\star \star *}$ \\
\hline Lo he realizado & $1,3^{\star \star \star}$ & $1,2^{*}$ & $4,9^{\star \star \star}$ & $3,8^{\star \star \star}$ & $2,0^{\star \star \star}$ & $1,6^{\star \star \star}$ \\
\hline \multicolumn{7}{|c|}{ P83st_e ¿Ha contactado a algún medio de comunicación? } \\
\hline Lo podría realizar & & & $1,7^{\star \star \star}$ & $1,1^{*}$ & $1,4^{\star \star \star}$ & $1,6^{\star \star \star}$ \\
\hline Lo he realizado & & $1,2^{*}$ & $2,2^{\star \star \star}$ & $2,0^{\star \star \star}$ & $2,5^{\star \star \star}$ & $2,9^{\star \star \star}$ \\
\hline \multicolumn{7}{|l|}{ Edad (Base $=16$ a 25 años) } \\
\hline 26-40 años & $0,9^{*}$ & & $1,3^{\star \star \star}$ & $1,2^{\star \star \star}$ & & \\
\hline
\end{tabular}




\begin{tabular}{|c|c|c|c|c|c|c|}
\hline \multicolumn{7}{|c|}{ Modelo Logit Multinominal } \\
\hline \multirow[b]{2}{*}{ Factores explicativos } & \multicolumn{2}{|c|}{ Votar } & \multirow{2}{*}{$\begin{array}{c}\text { Firmar una } \\
\text { petición } \\
\text { Lo he } \\
\text { realizado }\end{array}$} & \multirow{2}{*}{$\begin{array}{c}\text { Hacer cosas por el } \\
\text { barrio o comunidad } \\
\text { Trabajo }\end{array}$} & \multicolumn{2}{|c|}{$\begin{array}{l}\text { Mejorar el medio } \\
\text { ambiente }\end{array}$} \\
\hline & $\begin{array}{c}\text { Partido de } \\
\text { Gobierno }\end{array}$ & $\begin{array}{l}\text { Partido de } \\
\text { Oposición }\end{array}$ & & & Trabajo & Donar \\
\hline $41-60$ años & $0,8^{\star \star \star}$ & $0.8^{\star \star \star}$ & $1,4^{\star \star \star}$ & $1,3^{\star \star \star}$ & & $1,3^{\star \star}$ \\
\hline 61 y más & $0,8^{\star \star}$ & & $1,4^{\star \star \star}$ & & & \\
\hline \multicolumn{7}{|c|}{ Nivel educativo } \\
\hline Básica incompleta & & & $0,8^{*}$ & & & \\
\hline Básica completa & & & & & $1,2^{*}$ & \\
\hline $\begin{array}{l}\text { Secundaria, media o técnica } \\
\text { incompleta }\end{array}$ & & & & $0,7^{\star \star \star}$ & $1,3^{\star \star}$ & $1,4^{*}$ \\
\hline $\begin{array}{l}\text { Secundaria, media o técnica } \\
\text { completa }\end{array}$ & & $0,7^{\star \star}$ & $1,3^{*}$ & $0,7^{\star \star \star}$ & $1,5^{\star \star \star}$ & $1,6^{\star *}$ \\
\hline Superior incompleta & & $0,7^{\star \star}$ & $1,7^{\star \star \star}$ & $0,7^{\star \star}$ & $1,5^{\star \star \star}$ & $1,5^{\star}$ \\
\hline Superior completa & $0,6^{\star \star \star}$ & $0,7^{\star \star}$ & $2,0^{\star \star \star}$ & $0,6^{\star \star \star}$ & $1,5^{\star \star \star}$ & $1,8^{\star \star \star}$ \\
\hline \multicolumn{7}{|c|}{ P95st_a ¿Cómo se informa usted de los asuntos políticos? Familia (Base = No menciona) } \\
\hline Sí & & & & $1,1^{\star \star}$ & & $1,3^{\star \star \star}$ \\
\hline \multicolumn{7}{|c|}{ P95st_f ¿Cómo se informa usted de los asuntos políticos? Por diarios / revistas (Base = No menciona) } \\
\hline Sí & & & $1,1^{\star \star}$ & & $1,3^{\star \star \star}$ & \\
\hline \multicolumn{7}{|c|}{ P95st_g ¿Cómo se informa usted de los asuntos políticos? Internet (Base = No menciona) } \\
\hline Sí & $0,8^{\star *}$ & & $1,3^{\star \star \star}$ & & & $1,2^{\star}$ \\
\hline \multicolumn{7}{|c|}{ P95st_h ¿Cómo se informa usted de los asuntos políticos? TV (Base = No menciona) } \\
\hline Sí & & & $0,9^{*}$ & $1,1^{\star \star}$ & & \\
\hline \multicolumn{7}{|c|}{ P28st_c ¿Cuánta confianza tiene usted en los partidos políticos? (Base = Poco o nada de confianza) } \\
\hline Algo de confianza & $1,6^{\star \star \star}$ & $1,4^{\star \star \star}$ & & $1,2^{\star \star \star}$ & & $1,3^{\star \star \star}$ \\
\hline Mucha confianza & $2,1^{\star \star \star}$ & $1,6^{\star \star}$ & $1,4^{\star \star}$ & $1,4^{\star \star \star}$ & $1,3^{\star *}$ & \\
\hline \multicolumn{7}{|c|}{ P55st ¿Cuál diría usted que es su opinión sobre el funcionamiento de las instituciones públicas? (Base = Mal o muy mal) } \\
\hline Regular & $2,2^{\star \star \star}$ & $1,2^{\star \star}$ & $0,8^{\star \star \star}$ & $1,2^{\star *}$ & $1,3^{\star \star \star}$ & \\
\hline Bien o muy bien & $3,4^{\star \star \star}$ & $1,3^{\star *}$ & & & $1,2^{\star \star}$ & $1,4^{\star \star *}$ \\
\hline \multirow[t]{2}{*}{ Constante } & $0,2^{\star \star \star}$ & 0,7 & $0,1^{\star \star \star}$ & $0,2^{\star \star \star}$ & $0,3^{\star \star \star}$ & $0,0^{\star \star \star}$ \\
\hline & $(0,0)$ & $(0,2)$ & $(0,0)$ & $(0,0)$ & $(0,1)$ & $(0,0)$ \\
\hline Observaciones & \multicolumn{2}{|c|}{9,562} & 12,719 & 13,153 & \multicolumn{2}{|c|}{13,016} \\
\hline $\mathrm{R}^{2}$ & & & 0,1343 & .0927 & 0,1131 & \\
\hline
\end{tabular}

Error estándar en paréntesis *** $p<0,01,{ }^{\star \star} p<0,05,{ }^{\star} p<0,10$

Fuente: elaboración propia 
Los resultados del análisis estadístico muestran los siguientes patrones:

- En todos los casos (formas propositivas y directas de participación) los factores que más aumentan la propensión de los ciudadanos a participar son: i) formar parte de organizaciones (políticas y/o sociales). ii) Tener o haber tenido contacto con autoridades públicas y/o medios de comunicación. iii) Confiar en las instituciones de intermediación. iv) Tener una orientación ideológica no centrista, y especialmente considerarse de derecha o centro-derecha. Esto confirma por un lado la tesis de la movilización de recursos (asociada en alguna medida a la postura racional) según la cual, resolver los problemas de acción colectiva (al ser miembro de organizaciones dedicadas a la incidencia y/o intermediación) y desarrollar vínculos con los tomadores de decisión ofrece mayores oportunidades de incidir, y por tanto aumenta la propensión a participar. De allí que mayores niveles de confianza institucional estén relacionados positivamente con una mayor propensión a la participación. En tal sentido, las redes (que la literatura sugiere más clientelares en el caso de la participación directa, y más autónomas en el caso de las firmas y manifestaciones) y la capacidad de llegar hasta los tomadores de decisión parecen determinantes (Olson, 1965, Zaremberg, 2012).

- En tal sentido, quienes participan activando su voz (votando por la oposición y firmando peticiones ante las autoridades) y quienes mantienen su apoyo electoral a los gobiernos tienen perfiles similares. Esto pareciera contra-intuitivo, ya que cabría esperar que quienes activan su voz sean i) o bien más parecidos a quienes deciden salir o abstenerse de participar, o ii) diferentes de ambos, puesto que quien decide salir puede estar también satisfecho, por lo que no encuentra motivación para participar.

- Al concentrarnos en las diferencias, notamosquela participación electoral aumenta cuando:i) los ciudadanos consideran que el Gobierno representa los intereses del pueblo (si bien el efecto es -lógicamente- más fuerte en el caso de quienes votan por el Gobierno). ii) Hay una evaluación positiva de las instituciones públicas (siendo también más fuerte el efecto en los votantes oficialistas). iii) Se considera que el voto es la mejor forma de hacer que las cosas cambien. iv) Se cree que es posible confiar en las personas. v) Los ciudadanos tienen una orientación del centro a la derecha del espectro ideológico.

- Por su parte, si bien con diferencias entre ellos, quienes participan en acciones directas (trabajar por el barrio o la comunidad, y trabajar para mejorar el medioambiente) son más cínicos respecto del valor del régimen democrático. Sin embargo, al interior de este grupo quienes deciden trabajar por su comunidad son diferentes de aquellos que trabajan por fines sociales más amplios como la mejora del medioambiente. Los primeros tienen menor nivel educativo, se informan de los asuntos públicos por la televisión o la familia y tienden a confiar menos en las personas. Por su parte, sus contrapartes tienen mayores niveles educativos, suelen informarse de los asuntos políticos a través de periódicos o de Internet, presentan mayores índices de confianza (interpersonal e institucional) y evalúan de mejor manera el funcionamiento de las instituciones, acercándose a un perfil político más 'sofisticado', similar al de quienes activan su voz a través de formas no electorales como la firma de peticiones. Sin embargo, a diferencia de estos últimos, creen que el voto no es una herramienta útil para cambiar las cosas.

- En síntesis, y con un cierto grado de generalización, en América Latina nos encontramos, básicamente, con tres tipos de ciudadanos, en relación con su comportamiento político. i) En primer lugar, están aquellos que optan por la 'salida', ya sea de forma pasiva o activa. Los primeros no toman parte de ninguna forma 
de participación social, mientras que los activos, asumen formas de participación directa para resolver problemas puntuales (del barrio o la comunidad). En promedio, son menos educados, más desconfiados y críticos de las instituciones públicas, utilizan a la familia o a la televisión como medios principales de información política y pese a ser miembros de organizaciones sociales, parecen descreer de la posibilidad de cambiar las cosas. Su participación parece tener un tinte más particularista. Son quienes resuelven sus problemas en ausencia del Estado, o los que a través de sus contactos y/o de su participación en redes clientelares, entran en contacto con los intermediarios del sistema político formal para satisfacer sus necesidades. Su participación es tan racional como las otras, pero mucho más acotada y orientada, exclusivamente, a la satisfacción de sus necesidades. ii) En segundo lugar, están quienes activan su 'voz', pero básicamente a través de formas directas de participación. Este grupo, por lo general, considera que la participación en movimientos de protesta es una estrategia tan o más valiosa que el voto para cambiar las cosas, y deciden participar para cambiar el statu quo, tanto interpelando al Estado como incidiendo directamente en la mejora de ciertas condiciones. Estos ciudadanos tienen mayores niveles educativos, y se informan de los asuntos públicos a través de medios electrónicos o impresos, aunque difieren respecto de su cercanía con las instituciones. Quienes trabajan por la mejora del medioambiente de manera directa son menos críticos; tienden a confiary a evaluar mejor a las instituciones que quienes participan de acciones colectivas propositivas. iii) En tercer lugar, nos encontramos con aquellos que pese a todo consideran que el voto es la mejor estrategia para cambiar las cosas, y tienen niveles superiores de participación electoral, confianza personal e institucional y una evaluación positiva de la representatividad del Gobierno y las instituciones. Leales a la manera en que funciona el sistema, creen - al igual que quienes activan su vozque es posible incidir para que las cosas cambien, mientras que para quienes deciden salir o abstenerse de participar (perfiles cercanos a quienes trabajan para mejorar su barrio o comunidad) esta posibilidad parece vedada.

\section{Conclusiones}

Si bien este primer abordaje es exploratorio, parece un ejercicio útil para empezar a identificar perfiles ciudadanos, su vinculación con los asuntos públicos, sus comportamientos y el tipo de democracia que cabría esperar de su interacción con las instituciones representativas en América Latina.

En principio, para participar es necesario: i) tener información (ya sea que se obtenga a través de los medios de comunicación -televisión, radio redes sociales, periódicos-, o a través de la familia). ii) Tener una orientación ideológica definida. iii) Involucrarse en organizaciones. iv) Tomar la iniciativa de contactar a los funcionarios públicos o empresarios políticos responsables. Es decir, para participar no solo hay que querer (interés en la solución de problemas), sino que también hay que saber cómo (información) y hay que poder (tener vínculos institucionales a través de la membresía en organizaciones y/o tener acceso a funcionarios o tomadores de decisión que ayuden a resolver los problemas de acción colectiva típicos de la participación (Robinson, 2011).

Ahora bien, no todos los saberes y poderes son iguales. Si analizamos las formas de participación, los ciudadanos que participan de manera propositiva -ya sea a través del voto, o de la firma de peticiones - tienen un mayor nivel de confianza institucional. Esto contradice el supuesto de que la desconfianza aumenta la motivación 
de control de los ciudadanos. Por el contrario, confiar en las instituciones de intermediación como los partidos políticos parece un requisito para interpelar al sistema político -ya sea a través de firmas o del voto-y quienes están más decididos a activar su voz de protesta, también parecen ser más leales al régimen democrático, tanto respecto de sus actitudes como de su comportamiento político.

Por su parte, quienes participan más frecuentemente en 'acciones directas' (y que se concentran en países económicamente más desiguales y con muchos años de tradición autoritaria) tienen valores menos democráticos, consideran que las formas de participación no electoral son más adecuadas para cambiar las cosas, al considerar que estas no pueden cambiar, más allá de lo que uno haga.

Estos hallazgos vienen a reflejar dos realidades llamativas por sí mismas, pero mucho más interesantes por su carácter contrastante. Por un lado, se confirma el supuesto pluralista de que los más participativos son también los más pro-democráticos, si bien los más críticos sobre su funcionamiento. Los que más participan propositivamente, son quienes más esperan el retorno de su participación, y son quienes más esperan dicho retorno porque están mayormente integrados social y políticamente. Tienen mayor nivel educativo, están más definidos ideológicamente y confían más en las personas y en las instituciones. Es decir, son quienes probablemente activan su voz ante la caída en la calidad de los bienes públicos, pero también quienes expresan una mayor lealtad a la democracia. Por su parte, quienes participan en acciones directas tienen menor nivel educativo en términos relativos, confían menos en las personas, en el voto como forma de cambio social. Este fenómeno, lejos de constituir un puente entre participación y representación, puede ser un elemento que consolide y amplíe las desigualdades de acceso y ejercicio efectivo de los derechos, entre los ciudadanos más y menos propensos a participar. Dicho de otra forma, son los menos privilegiados en términos sociales quienes reducen su participación al ámbito comunitario (quienes optan por la 'salida' en términos de Hirschman), demostrando, además, un menor apego a la democracia representativa, de la cual probablemente, ya no esperen demasiado.

\section{Referencias}

1. Aguilar, L. (1992). Estudios de política pública. México: Miguel Ángel Porrúa.

2. Almond, G., \& Verba, S. (1959). The Civic Culture. Princeton: Princeton University Press.

3. Altman, D. (2010). Direct Democracy Worldwide. New York: Cambridge University Press.

4. Anduiza, E., \& Bosch, A. (2004). Comportamiento político y electoral. Madrid: Ariel.

5. Avritzer, L. (2010). Las instituciones participativas en el Brasil democrático. Xalapa: Universidad Veracruzana.

6. Bandura, A. (1986). Social Foundations of Thought and Action: A Social-Cognitive Theory. Englewood Cliffs, NJ: Prentice Hall.

7. Barnes, S., \& Kaase, M. (1979). Political Action. Mass Participation in Five Western Democracies. Londres: Sage.

8. Best, S. \& Krueger, B. (2005): Analyzing the Representativeness of Internet Political Participation. Political Behaviour, 27(2), 183-216.

9. Brusco, V., Nazareno, M., \& Stokes, S. (2004). Vote Buying in Argentina. Latin American Research Review, 39(2), 66-88.

10. Buchanan, J., \& Tullock, G. (1980). El cálculo del consenso. Madrid: Espasa. 
11. Castillo, J. (2008). Trayectorias de participación política de la juventud europea: ¿Efectos de cohorte o efectos de ciclo vital? Revista de Estudios de Juventud, 81, 68-94.

12. Cleary, M., \& Stokes, S. (2006). Democracy and the Culture of Skepticism: Political Trust in Argentina and Mexico. New York: Russell Sage Foundation.

13. Dahl, R. (1989). Poliarquía: participación y oposición. Madrid: Tecnos.

14. De Sousa, B. (2004) (Coord.). Democratizar la democracia. Los caminos de la democracia participativa. México D.F.: Fondo de Cultura Económica.

15. Easton, D. (1953). The Political System. New York: Alfred A. Knopf, Inc.

16. Franklin, M., Lyons, P., \& Marsh, M. (2004). Generational Basis of Turnout Decline in Established Democracies. Acta Politica, 39, 115-151.

17. Hawkins, K. (2010). Who Mobilize? Participatory Democracy in Chavez' Bolivarian Revolution. Latin American Politics and Society, 52(3), 31-66.

18. Henn, M., Weinstein M., \& Wring, D. (2002). A Generation Apart? Youth and Political Participation in Britain. British Journal of Politics and International Relations, 4, 167-192.

19. Hirschman, A. (1977). Salida, voz y lealtad. México: Fondo de Cultura Económica.

20. Hirschman, A. (1986): Interés privado y acción colectiva. México: Fondo de Cultura Económica.

21. Granato, J., Inglehart, R., \& Leblang, D. (1996). Cultural Values, Stable Democracy and Economic Development: A Replay. American Journal of Political Science, 40(3), 680-696.

22. Gunitzky, S. (2013). Lost in the Gray Zone. Competing Measures of Democracy in the Former Soviet Republics. En A. Cooley \& J. Snyder (Eds.). Ranking the World. Cambridge University Press.

23. Irving, L. (2006). Challenges and Strategies for Democratic Participation. Recuperado de www. beyondintractability.org/print/2672

24. Isunza, E., \& Gurza, A. (2010). Precisiones conceptuales para el debate contemporáneo sobre la innovación democrática: participación, controles sociales y representación. En E. Vera \& A. Gurza (Coords.). La innovación democrática en América Latina. Tramas y nudos de la representación, la participación y el control social. México: CIESAS-Universidad Veracruzana.

25. Lasswell, R. (1992). La orientación hacia las políticas. En L. Aguilar (Ed.). El estudio de las políticas. México: Miguel Ángel Porrúa.

26. Levine, D., \& Molina, J. (2007). La calidad de la democracia en América Latina. Una visión comparada. Revista América Latina Hoy, 45, 17-46.

27. Manin, B. (1998). Los principios del gobierno representativo. Madrid: Alianza Editorial.

28. Milbraith, L., \& Goel, M. (1965): Political Participation: How and Why Do People Get Involved in Politics? Chicago: Rand McNally and Company.

29. Olson, M. (1965). The Logic of Collective Action. Public Goods and the Theory of Groups. Cambridge: Harvard University Press.

30. Offe, C. (2006). Democracy, Disaffection and Institutions: Some Neo-Tocquevillean Speculations. En M. Torcal \& J. Montero (Eds.). Political Disaffection in Contemporary Democracy: Social Capital, Institutions and Politics. Londres: Routledge.

31. Parés, M. (Coord.) (2009). Participación y calidad democrática. Barcelona: Ariel.

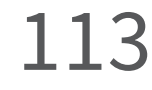


32. Peruzzotti, E. \& Smulowitz, C. (Eds.) (2002). Accountability Societal: Controlando la política. Ciudadanos y medios en las democracias latinoamericanas. Buenos Aires: Temas.

33. Pitkin, H. (1985). El concepto de representación. Madrid: Centro de Estudios Constitucionales.

34. Putnam, R. (1993). Making Democracy Work. Princeton, NJ: Princeton University Press.

35. Robinson, J. (2011). La economía política de la redistribución. En N. Lustig \& F. López (Comp.). La disminución de la desigualdad en América Latina. México: Fondo de Cultura Económica.

36. Ruedin, D. (2007). Testing Milbrath's 1965 Framework of Political Participation: Institutions and Social Capital. Contemporary Issues and Ideas in Social Sciences, 3(3). Recuperado de http://journal.ciiss.net/ index.php/ciiss/article/view/43/50

37. Schedler, A. (2007). ¿Qué es la rendición de cuentas? México DF: Cuadernos de Transparencia No. 3, Instituto Federal de Acceso a la Información.

38. Schmitter, P. \& Lynn, K. (1991). Democracy: What is and is not? Journal of Democracy, 2(3), 75-88.

39. Uslaner, E. (2002). Civic Engagement in America: Why People Participate in Political and Social Life. Recuperado de http://www.politicipublice.ro/uploads/civic_engagement_in_america.pdf

40. Vargas, S. (2011). Evaluando la participación social: Democracia y políticas públicas. Revista Mexicana de Sociología, 73(1), 105-137.

41. Vecchione, M. \& Vittorio, G. (2009). Personality Determinants of Political Participation: The Contribution of Traits and Self-Efficacy Beliefs. Personality and Individual Differences, 46(4), 487-492.

42. Verba, S., Lehman, K., \& Brady, H. (1995). Voice and Equality: Civic Voluntarism in American Politics. Cambridge: Harvard University Press.

43. Welp, Y. (Coord.) (2011). Caleidoscopio de la innovación democrática en América Latina. México: FLACSO.

44. Zaremberg, G. (2012). Nuevas instituciones de democracia participativa en América Latina. La voz y sus consecuencias. México D.F.: FLACSO. 